\title{
Canlı Donör Karaciğer Naklinde Safra Yolu Varyasyonlarının Biliyer Komplikasyonlara Etkisi
}

\author{
Hikmet AKTAŞ
}

Acıbadem Bursa Hastanesi, Organ Nakli Bölümü, Bursa.

\begin{abstract}
ÖZET
Canlı donör karaciğer nakillerinde, donör safra yollarının bölünmesi donör hepatektominin en kritik aşamalardan biridir. Bu çalıșmada, merkezimizde să̆ lobektomi uygulanan canlı donörlerin safra yolu varyasyonlarının belirlenmesi ve bu varyasyonların postoperatif alıcı safra komplikasyonları ile ilişsisi araştııılmışıı. Ocak 2016 ile Ocak 2018 arasında yapılan canlı donör hepatektomi olgularının verileri retrospektif olarak incelendi. Araştırmaya sağ lobektomi uygulanan donörler (229 olgu) ve minimum 2 yıl takip süresi olan alıcıları dahil edildi. Re-trasnplantasyon yapılan ve primer biliyer patoloji nedeniyle karaciğer nakli uygulanan alıcılar çalışma dışı bırakıldı. Huang sınıflamasına göre en sık tespit edilen tip A1 (\%45) idi. Donörler, greft safra ağzı tek, çift ve üç adet olanlar şeklinde sırasıyla grup A,B ve C olmak üzere üç gruba ayrılarak postoperatif komplikasyonlar karşılaştırıldı. Group A, B ve C sırasıyla $123(\% 53,7), 94(\% 41)$ ve $12(\% 5,2)$ olgudan oluştu. 36 hastada safra komplikasyonu (darlık, kaçak) tespit edildi $(\% 15,7)$. Gruplar arasında yapılan karşılaştırmada safra komplikasyonu açısından anlamlı fark tespit edilmedi $(\mathrm{p}>0.05)$. Pre-perioperatif safra yolu değerlendirmesinin etkin yapılması ve uygun cerrahi stratejinin belirlenmesi durumunda greft safra ağzı sayısının alıcı safra komplikasyonları açısından kötü prognostik etkisi yoktur.
\end{abstract}

Anahtar Kelimeler: Karaciğer nakli. Safra anatomisi. Canlı donör. Safra komplikasyonu.

Effect of Biliary Tract Variations on Biliary Complications in Living Donor Liver Transplantation

\begin{abstract}
Dividing bile ducts during donor hepatectomy is one of the critical step of the living donor liver transplantation. To determine the biliary anatomy of graft who underwent right lobe donor hepatectomy in our center and to evaluate effect of biliary variations on recipient biliary complications was the main purpose of this study. The data of donors who were performed right donor hepatectomy between January 2016 and January 2018, were analyzed retrospectively. Two hundred twenty-nine patients who were carried out right lobe donor hepatectomy and the recipients of these donors who had at least two years follow-up were included in the study. Recipients who underwent liver transplantation due to primary biliary pathology and re-transplanted recipients were excluded. The most detected biliary type was A1 (45\%) according to Huang classification. Donors were divided into three groups in related to number of bile duct orifice as group A, B and C which contains one, two and three bile duct orifices, respectively and postoperative biliary complications were compared between these groups. There were 123 (53,7\%), 94 (41\%) and 12 (5,2\%) patients in Group A, B and C, respectively. Thirty-six recipients (15,7\%) experienced biliary complications. There was no statistical difference between groups associated with biliary complications $(\mathrm{p}>0.05)$. The number of greft bile orifice has no effect on recipient biliary complications wether, if the pre-perioperative assessment of bile anatomy is investigated effectively and the surgical strategy is decided properly.
\end{abstract}

Key Words: Liver transplantation. Biliary anatomy. Living donor. Biliary complications.

Geliş Tarihi: 02.Nisan.2021

Kabul Tarihi: 14.Haziran.2021

8. Uludağ Genel Cerrahide Güncel Yaklaşımlar Toplantısı (17 Mart 2019 Uludağ/Bursa) sözlü biliri olarak sunulmuştur.

Dr. Hikmet AKTAS

Acıbadem Bursa Hastanesi, Organ Nakli Bölümü, Bursa Tel.: 05322077660

E-posta:drhikmetaktas@hotmail.com

Yazarların ORCID ID Bilgisi:

Hikmet AKTAŞ: 0000-0001-7943-0633
Son iki dekadda, safra yollarının damarsal beslenmesi ve varyasyonları daha iyi anlaşılmasına ve cerrahi tekniklerde gelişim gösterilmesine rağmen, safra komplikasyonları canlı donör karaciğer nakli (CDKN) sonrası en önemli morbidite ve mortalite sebebi olmaya devam etmektedir. Anastomoz darlığı başta olmak üzere safra komplikasyonlarının CDKN sonrası insidansının (\%5.3-\%40.6) yüksek olmasının nedenleri olarak öne çıkan faktörler, çok sayıda ve farklı çapta safra yolu ağzı varlığı, safra ağacı beslenmesinin bozulması ve cerrahi yetersizliklerdir ${ }^{1-5}$.

Safra yolları anatomisinin sınıflandırılması, endoskopik retrograd kolanjiografi (ERK), intraoperatif kolanjiografi (İK), bilgisayarlı tomografi kolanjiografi (BT-K) ve magnetik rezonans kolanjiografi (MRK) 
bulgularına dayandırılarak yapılmıștır ${ }^{6-8}$. Literatürde en sık kullanılan Huang sinıflaması ${ }^{6}$, sağ posterior hepatik safra yolunun (SPSY) ana safra yoluna (ASY) dökülme şekline göre tanımlanmıştır. Ancak daha sonra yapılan çalışmalar, varyasyonların insidansları açısından farklı irklarda ve farklı coğrafyalarda önemli farklar olduğunu ortaya koymuştur ${ }^{7-9}$. Türkiye'den yapılan iki farklı çalışmada ${ }^{10,11}$ da Huang'ın bildiridiği varyasyon insidanslarından farklı oranlar ortaya konulmuştur.

Bu çalışmada, CDKN sayısı açısından yüksek volümlü olan merkezimizde, canlı donör safra yolu varyasyonlarının ortaya konulması ve bu varyasyonların alıcı safra komplikasyonlarına olan etkisinin ortaya konulması amaçlandı. Donöre ait faktörler olarak yaş ve vücut kitle indeksi (VKI) ve safra anastomozu tekniğinin safra komplikasyonlarına etkisi ayrıca incelendi. Çalışmanın, özellikle bölgesel safra yolu haritası çıkarılmasına katkı sunması öncellenmiştir.

\section{Gereç ve Yöntem}

Çalışma retrospektif olarak dizayn edildi ve lokal etik kurul onayı alındı (ATADEK:2020-15/9). Ocak 2008 ile Ocak 2018 arasında yapılan sağ lob donör hepatektomi olguları ve bu donörlere ait alıcılardan en az iki y1llık takip süresi olanlar çalıșmaya dahil edildi. Karaciğer re-transplantasyon uygulanan ve primer sklerozan kolanjit, primer biliyer siroz gibi esas olarak safra yolu patolojisine bağlı olarak karaciğer nakli uygulanan alıcılar (ve donörleri) çalışma dışı bırakıldı.

Merkezimizde, canlı donör safra anatomisi değerlendirilmesi, rutin olarak preoperatif yapılan MR-K ve peroperatif yapılan İOK bulguları ile değerlendirilmektedir. Peroperatif alıcı safra yolu bulguları da dahil edilerek Roux-en-Y duktojejunostomi veya uçuca safra yolu anastomozu kararı verilmektedir. Grefte ait değerlendirlen bulgular, safra ağızı sayısı ve çoklu ise birbirine mesafesidir. Alıcıya ait bulgular ise: alıcı safra yolu mukozasının kanlanmasının durumu, çevre dokularının disseksiyon sırasında korunmuş olup olmadığı, greft safra yolu sayısına göre alıcı safra yollarının sayısı ve çap uyumsuzluğu olup olmadığıdır. Çoklu safra anastomoz yapıldığında ve kanlanmaya dair şüphe içeren durumlarda safra yollarına kateter konulmaktadır. Bu kateter ilk 4 hafta açık takip edilip sonra kapatılmaktadır. Nakil sonrası üçüncü ayda kontrol kolanjiografi çekildikten sonra kateter çekilmektedir. Bu kolanjiografide darlık tespit edildiği durumlarda, uçuca safra anastomozu yapılan hastalar ERK işlemine yönlendirilmektedir. Duktojejunostomi hastalarında anastomozun fizyolojisi nedeniyle perkütan transhepatik kolanjiografi (PTK) yöntemi kullanilmaktadir.

Safra komplikasyonu tanısı, klinik olarak kolanjit ve peritonit bulguları gelişmesi, drenlerde safra varlığı ve laboratuar olarak bilüribin ve alkalen fosfataz değerlerinde yükselme olması durumunda konulmaktadır. Tanı, MR-K ile doğrulanmaktadır. Klinik olarak kontrol altındaki minimal kaçaklarda ilk tercih olarak konservatif kalınmaktadır. Ancak, ilerleyen ve baştan major olan kaçaklarda ERK ile safra stenti yerleştirilmesi ilk tercih edilen invaziv tedavi yöntemidir. Başarısız olunan durumlarda, PTK ile drenaj kateteri yerleştirilmektedir.

Donörlere ait demografik veriler, safra yolu anatomisi bulguları ve postoperatif safra komplikasyonlarına ait veriler retrospektif incelendi. Donörlere ait safra anatomisi Huang yöntemine göre sınıflandırıldı. Sınıflandırmada İOK bulguları esas alındı. Donörler, grefte ait safra yolu ağzı sayısına göre 3 gruba ayrıldı. Grup A tek safra yolu, grup B iki safra yolu ve grup B üç safra yolu ağzına sahip greftleri kapsiyordu.

Alıcılara ait safra darlığı ve kaçağı komplikasyonları retrospektif olarak incelendi. Bu komplikasyonlar birlikte ve ayrı ayrı donör referanslı oluşturulan üç gruba göre istatiksel olarak karşılaştırıldı.

\section{Istatistiksel Analiz}

İstatistiksel analizler Statistical Package for the Social Sciences (SPSS) version 22.0 (SPSS Inc., Chicago, IL, USA) programında yapılmıştır. Tanımlayıcı veriler, ortalama (min-max) ve yüzde (\%) olarak tanımlandı. Kategorik değişkenler frekans (n) ve yüzde (\%) değerleri ile verilmiş olup, karşılaştırmalarında Fisher'ın exact chi-square testi kullanılmıştır. Anlamlılık düzeyi $\mathrm{p}<0,05$ olarak alınmıştır.

\section{Bulgular}

Kriterlere uyan 229 donör ve alıcıları çalışmaya dahil edildi. 131 donör erkek $(\% 57,2)$ ve 98 donör kadın $(\% 42,8)$ idi. Yaş ortalaması 31,6 (18-57 yaş) idi.

Huang sinıflandırmasına göre, A1 tipi en çok tespit edilen (103, \%45) safra varyasyonu iken, A5 en nadir görülen $(2, \% 0,9)$ varyasyon idi. Grup A, B ve C sırasiyla $123(\% 53,7), 94(\% 41)$ ve $12(\% 5,3)$ donörden oluşuyordu. Safra anastomozlarının altı tanesi $(\% 2,6)$ duktojejunostomi ve diğerleri $(223, \% 97,4)$ uç-uca anastomoz şeklinde yapıldı.

Donörlerin sadece yedisinde safra kaçağı tespit edilirken (\%3), hiçbir donörde safra yolu darlığı izlenmedi. $\mathrm{Bu} 7$ olgudan dördünde safra kaçağı postoperatif ilk 1 haftada spontan kapandi ve takiplerde problem yaşanmadi. 3 hastada ise girişimsel radyoloji tarafindan drenaj kateteri konuldu ve 1 ay sonra safra kaçağ 1 spontan kapandı ve drenaj kateteri çekildi. Hiçbir donörde ERK gerekmedi. Donörlere ait safra yolu verileri Tablo I’de listelendi. 


\section{Donör Safra Anatomisi ve Safra Komplikasyonları}

Tablo I. Donör safra anatomisi bulguları

\begin{tabular}{|l|ll|}
\hline & Donör sayısı (n:229) \\
\hline Huang sınıflamasına göre: & 103 & $(\% 45)$ \\
Tip A1 & 51 & $(\% 22.3)$ \\
Tip A2 & 63 & $(\% 27.5)$ \\
Tip A3 & 10 & $(\% 4.3)$ \\
Tip A4 & 2 & $(0,9)$ \\
Tip A5 & & \\
\hline Greft safra ağzına göre: & 123 & $(\% 53.7)$ \\
Grup A: tek ağız & 94 & $(\% 41)$ \\
Grup B: çift ağız & 12 & $(\% 5.3)$ \\
Grup C: üç ağız & & \\
\hline
\end{tabular}

Alıcılarda toplam safra komplikasyonu oranı \%15,7 $(36 / 229)$ idi. Safra darlığı ve kaçağı sayısı sırasıyla 30 $(\% 83,3)$ ve $6(\% 6,7)$ idi. Safra anatomisi ile alıcı safra komplikasyonları arasında, Huang Tip 2 dominans1 olmak üzere, istatistiksel anlamlı ilişki mevcuttu $(\mathrm{p}<0.001)$ (Tablo II). Greft safra sayısı ile alıcı safra komplikasyonları arasında istatistiksel anlamlı ilişki saptanmadi $(\mathrm{p}=0.121)$ (Tablo III).

Tablo II. Safra anatomisi ve safra komplikasyonları

\begin{tabular}{|c|c|c|c|c|c|c|c|c|}
\hline & & \multicolumn{6}{|c|}{ Alıcı Safra Komplikasyonu } & \multirow{3}{*}{ Anlamlılık (p } \\
\hline & & \multicolumn{2}{|c|}{ Yok } & \multicolumn{2}{|c|}{ Darlık } & \multicolumn{2}{|c|}{ Kaçak } & \\
\hline & & $\mathrm{n}$ & $\%$ & $\mathrm{n}$ & $\%$ & $\mathrm{n}$ & $\%$ & \\
\hline \multirow{5}{*}{ Huang Tip } & 1 & 100 & 51,8 & 2 & 6,7 & 1 & 16,7 & \multirow{5}{*}{$<0,001$} \\
\hline & 2 & 39 & 20,2 & 10 & 33,3 & 3 & 50 & \\
\hline & 3 & 48 & 24,9 & 13 & 43,3 & 2 & 33,3 & \\
\hline & 4 & 6 & 3,1 & 3 & 10 & 0 & 0 & \\
\hline & 5 & 0 & 0 & 2 & 6,7 & 0 & 0 & \\
\hline
\end{tabular}

Tablo III. Greft safra sayısı ve safra komplikasyonları

\begin{tabular}{|c|c|c|c|c|c|c|c|c|}
\hline & & \multicolumn{6}{|c|}{ Alıcı Safra Komplikasyonu } & \multirow{3}{*}{ Anlamlılık (p) } \\
\hline & & \multicolumn{2}{|c|}{ Yok } & \multicolumn{2}{|c|}{ Darlık } & \multicolumn{2}{|c|}{ Kaçak } & \\
\hline & & $n$ & $\%$ & $\mathrm{n}$ & $\%$ & $n$ & $\%$ & \\
\hline \multirow{3}{*}{\multicolumn{2}{|c|}{$\begin{array}{ll} & 1 \\
\text { Safra } & \text { Yolu } \\
\text { Sayısı } & 2 \\
& 3\end{array}$}} & 109 & 56,5 & 12 & 40 & 2 & 33,3 & \\
\hline & & 76 & 39,4 & 15 & 50 & 3 & 50 & 0,121 \\
\hline & & 8 & 4,1 & 3 & 10 & 1 & 16,7 & \\
\hline \multicolumn{9}{|c|}{ Fisher's Exact Test, $p<0,05$} \\
\hline
\end{tabular}

\section{Tartışma ve Sonuç}

Donör havuzunun darlığı nedeniyle CDKN içeren büyük serilerin çoğunluğu Asya ülkeleri tarafından yayımlanmaktadır. Dolayısıyla, safra anatomisine dair veriler çoğunlukla sarı ırka ait verilerden oluşmaktadır. Çin, Japonya ve Kore kaynaklı yayınlarda bildirilen safra anatomisi subtiplerinin oranları Huang tarafindan tanımlanan oranlarla benzerlik göstermektedir ${ }^{12}$. Ayrı- ca, bir başka literatür değerlendirmesinde ${ }^{9}$, Almanya hariç, Avrupa ve Amerika ülkelerinden bildirilen oranların da benzer olduğu gösterilmiştir. Sonuç olarak, Huang tip A1 safra anatomisi baskın safra anatomisi olarak görülmektedir. Bizim çalışmamızda da en yaygın tip A1(\%45) idi. Ancak literatürdeki bildirilen ${ }^{6,8,9}$ insidanslara (\%55-76) göre daha düşük oranda idi. Dikkat çekici esas nokta, Türkiye'den yapılan üç yayında ${ }^{10,11,17}$ tipik (normal) safra anatomisi olarak tarif edilen Huang A1 tipi insidansı \%61, \%76 ve $\% 79,4$ olarak bildirilmiştir. Bizim çalışmamızda bu oranın düşük olması, bahsedilen üç çalışmadan farklı olarak bizim donör grubumuzda \%21 oranında uluslararası hasta varlığı olması ile açıklanabilir.

CDKN sonuçlarını bildiren yayımların büyük çoğunluğunda uçuca anastomoz en çok uygulanan (\%70-90) safra rekonstrüksiyonudur ${ }^{14,15}$. Bizim serimizde bu oran \%97,4 olarak belirgin yüksektir. Ancak duktojejunostominin rutin tercih edildiği primer safra yolu patolojileri nedeniyle yapılan nakiller çalışma dişı bırakıldığı için oranın daha yüksek çıktığını söyleyebiliriz. Safra komplikasyonlarını değerlendiren CDKN içeren yayınlar incelendiğinde, ilk çalışmalarda ${ }^{1-4}$ donör safra sayısı safra komplikasyonları için risk faktörü olarak tanımlanırken, yakın zamanda yayımlanan çalışmalarda ${ }^{16,18}$ greft safra yolu sayısı ile safra komplikasyonları arasında ilişki bulunmazken, risk faktörü olarak safra yolu beslenmesini etkileyen faktörler öne çıkmaktadır. Bizim serimizde de safra yolu sayısı ve safra komplikasyonları arasında anlamlı bir ilişki bulunmamıştır. Bizim çalışmamızda dikkat çeken bir diğer nokta, Karakaş ve ark. ${ }^{8}$ nın dikkat çektiği gibi, zaman zaman preoperatif sınıflama ile uyumsuz sayıda greft safra ağzı orataya çıkabilmektedir. Çalışmamizda Huang tip A2 olan 12 hasta ve A3 olan 8 hastanın safra ağız sayısı beklenenin aksine tek ağız şeklinde (çift-bileşik safra yolu) elde edilmiştir. Çalışmaların Huang sınıflaması değil de safra ağzı sayısına göre yapılması bununla ilişkili görünmektedir. Bizim çalışmamızda Huang sınıflaması ile safra komplikasyonları arasında istatistiksel olarak anlamlı ilişki bulunmuştur. Özellikle Huang Tip 2 ve 3 ile alıcı safra yolu darlığı arasında anlamlı ilişki bulunmuştur. Ancak, greft safra ağzı sayısı ile safra komplikasyonları arasında benzer bir iliş̧i bulunmadı. Bu durum, Huang sınıflaması ile split sonrası çıkan safra ağzı sayısının örtüşmemesi ve hatta cerrahi olarak ek tanımlamalar (çift bileşik, gözlük vs) yapılan koşullarda anastomoz sayısı tek olsa da safra ağzı sayısının çift olması ile açıklanabilir. Bizim serimizde de, Huang tip 2 ve 3 olan ve normalde çift safra ağzı çıkmış varsayılması gereken 20 hasta bahsedilen tanımlamalarla tek ağız olarak kabul edilmiştir.

Safra komplikasyonu, CDKN sonrası hala en ciddi problemlerden birini oluşturmaktadır. Cerrahi merkezlerin deneyimi, canlı donör seçiminde safra yolu anatomisini bir kriter olarak belirlemede temel faktör 
olarak görülmektedir. CDKN açısından yüksek volümlü merkezlerde, safra yolu sayısı safra komplikasyonları açısından anlamlı bir fark ortaya çıkartmamaktadir.

Etik Kurul Onay Bilgisi:

Onaylayan Kurul: Acıbadem Mehmet Ali Aydınlar Üniversitesi Tibbi Araștırmalar Değerlendirme Kurulu

Onay Tarihi: 09.07.2020

Karar No: 2020-15/9

\section{Araștırmacı Katkı Beyanı:}

Fikir ve tasarım: H.A.; Veri toplama ve işleme: H.A.; Analiz ve verilerin yorumlanması: H.A.; Makalenin önemli bölümlerinin yazılması: H.A. tarafından yapılmışıtır.

Destek ve Teşekkür Beyanı:

Doç.Dr. Ali Özer'e verilerin yorumlanması sürecinde verdiği destek için teșekkür ederim.

Çıkar Çatışması Beyanı:

Makale yazarının çıkar çatışması beyanı yoktur.

\section{Kaynaklar}

1. Gondolesi GE, Varotti G, Florman, et al. Biliary Complications in 96 Consecutive Right Lobe Living Donor Transplant Recipients. Transplantation. 2004;77(12):1842-8.

2. Takatsuki M, Eguchi S, Kawashita Y, Kanematsu T. Biliary complications in recipients of living-donor liver transplantation. J Hepatobiliary Pancreat Surg. 2006;13:497-501.

3. Yazumi S, Chiba T. Biliary complications after a right-lobe living donor liver transplantation. J Gastroenterol. 2005;40:861-5.

4. Yaprak O, Dayangac M, Akyildiz M, et al. Biliary complications after right lobe living donor liver transplantation: a singlecentre experience. HPB (Oxford). 2012;14(1):49-53.

5. Ye S, Dong JH, Duan WD, Ji WB, Liang YR. The Preliminary Study on Procurement Biliary Convergence from Donors with Complicated Bile Duct Variant in Emergency Right Lobe Living Donor Liver Transplantation. J Clin Exp Hepatol. 2017;7:33-41.
6. Huang TL, Cheng YF, Chen CL, Chen TY, Lee TY. Variants of the bile ducts: clinical application in the potential donor of living-related hepatic transplantation. Transpl Proc. 1996;128:1669-70.

7. Nakamura T, Tanaka K, Kiuchi T, et al. Anatomical variations and surgical strategies in right lobe living donor liver transplantation: lessons from 120 cases. Transplantation. 2002;73:18961903

8. Varotti G, Gondolesi GE, Goldman J, et al. Anatomic variations in right liver living donors. J Am Coll Surg. 2004; 198:577-82.

9. Chaib E, Kanas AF, Galvão FHF, D'Albuquerque LAC. Bile duct confluence: anatomic variations and its classification. Surg Radiol Anat. 2014;36(2):105-9.

10. Karakas HM, Celik T, Alicioglu B. Bile duct anatomy of the Anatolian Caucasian population: Huang classification revisited. Surg Radiol Anat. 2008;30:539-45.

11. Uysal F, Obuz F, Uçar A, Seçil M, Igci E, Dicle O. Anatomic variations of the intrahepatic bile ducts: analysis of magnetic resonance cholangiopancreatography in 1011 consecutive patients. Digestion. 2014;89(3):194-200.

12. Chittapuram S, Ramesh Babu, Malay Sharma. Biliary tract anatomy and its relationship with venous drainage. J Clin Exp Hepatol. 2014;4 (Suppl 1):18-26.

13. Dusunceli E, Erden A, Erden I. Anatomic variations of the bile ducts: MRCP findings. Tani Girisim Radyol. 2004;10:296-303

14. Chok KSH, Lo CM. Systematic review and meta-analysis of studies of biliary reconstruction in adult living donor liver transplantation. ANZ J Surg. 2017;78:121-5.

15. Azzam AZ, Tanaka K. Biliary complications after living donor liver transplantation: A retrospective analysis of the Kyoto experience 1999-2004. Indian J Gastroenterol. 2017;36(4):296304.

16. Mathew JS, Manikandan K, Kumar KYS, et al. Biliary complications among live donors following live donor liver transplantation. The Surgeon. 2018;16:214-9.

17. Tezcaner T, Dinç N, Karakayalı FY et al. Effect of Right Posterior Bile Duct Anatomy on Biliary Complications in Patients Undergoing Right Lobe Living Donor Liver Transplant. Exp Clin Transplant. 2019;17(6):759-67.

18. Nakamura $T$, Iida $T$, Ushigome $H$, et al. Risk Factors and Management for Biliary Complications Following Adult Living-Donor Liver Transplantation. Ann Transplant. 2017; 22:671-6 